\title{
Electrophysiologic recordings in a patient with a discrete unilateral thalamic infarction
}

\author{
EJ HAMMOND, * BJ WILDER, * WE BALLINGER, JR $\dagger$ \\ From the Neurology Service* and Laboratory Service (Neuropathology), $\dagger$ Veterans Administration Medical \\ Center, Gainesville, Florida, USA
}

SUMMARY The electroencephalogram, and somatosensory and auditory evoked potentials were recorded from a patient, who, at necropsy, showed a restricted unilateral thalamic infarct involving predominantly the anterior and lateral thalamus. The electroencephalogram showed distinct monomorphic delta activity and a suppression of the alpha rhythm over the side of the lesion. Short latency somatosensory evoked potentials were present bilaterally; mid-latency somatosensory evoked potentials were absent ipsilateral to the lesion. Both mid-latency and long latency auditory evoked potentials were normal.

Stimulus pulses to the median nerve evoke characteristic potentials over the contralateral somatosensory cortex, consisting of a negative potential occurring with a latency of about $20 \mathrm{~ms}$ followed by one or more positive potentials. ${ }^{1}$ The $\mathrm{N} 20$ component is thought to represent the incoming volley at the primary somatosensory cortex $;^{1}$ some have recently argued for a thalamic origin for this potential. ${ }^{2} \mathrm{~A}$ negative potential at $33 \mathrm{~ms}$ latency is also recorded precentrally. ${ }^{3}$ With appropriate technique three shorter latency somatosensory evoked potentials can also be recorded from the scalp. Topographic mapping studies suggest that the first wave is generated at the level of the brachial plexus, the second from within the spinal cord and the third from a level above the medulla. ${ }^{4-6}$ The determination of the actual neural sources of these components awaits correlation of electrophysiologic recordings with verified subcortical lesions. Aside from findings in one patient described by Mauguière and Courjon, ${ }^{7}$ such correlations relevant to this problem have been limited to computed tomography findings (CT). We present here evoked potential, electroencephalographic and detailed neuropathologic findings in a patient with a discrete unilateral thalamic infarction, who presented with distinct electrophysiologic asymmetries.

Address for reprint requests: EJ Hammond, PhD, Neurology Service, VA Medical Center, Gainesville, F1 32602, USA.

Received 27 February 1982. Accepted 18 March 1982

\section{Methods}

Case report The patient was a 66-year-old male with a long history of hypertension, who, while working in the sun, became weak and collapsed. At the time of hospital admission examination showed confusion, a mixed transcortical aphasia, right hemiparesis and right hemisensory deficit. Ten days after admission neurological examination revealed a mild right facial weakness, a right hemiparesis with arm weaker than leg and distal muscles weaker than proximal muscles, and a right hemisensory deficit for graphaesthesia and stereognosis, vibration, pin and touch sensation. Grasp, suck, and root responses were present. Reflexes were enhanced and the Babinski sign was present bilaterally. Cerebellar examination was within normal limits. CT revealed a radiolucent area confined to the left thalamus. Three days after electrophysiological recordings were made the patient suffered cardiorespiratory arrest from which he could not be resuscitated.

Postmortem examination of the brain revealed atherosclerosis involving the major cerebral arteries with marked involvement of the left posterior cerebral artery just distal to the junction of the left posterior communicating artery. A thrombus was found in this region which extended for approximately $1.0 \mathrm{~cm}$. This thrombus apparently occluded perforating vessels which serve portions of the thalamus. Horizontal sections of the brain demonstrated an area of infarction involving the lateral portions of the left thalamus (fig 1). This infarct involved all of the ventral anterior, ventral lateral and lateral posterior nuclei. It also involved the anterior portion of the pulvinar and the lateral portion of the dorsomedial nucleus of the thalamus. The anterior nucleus of the thalamus was spared as well as the majority of the dorsomedial nucleus. The lesion extended rostally to involve a small region of the prerubal radiations. Involvement of the internal capsule on the left was very minimal 


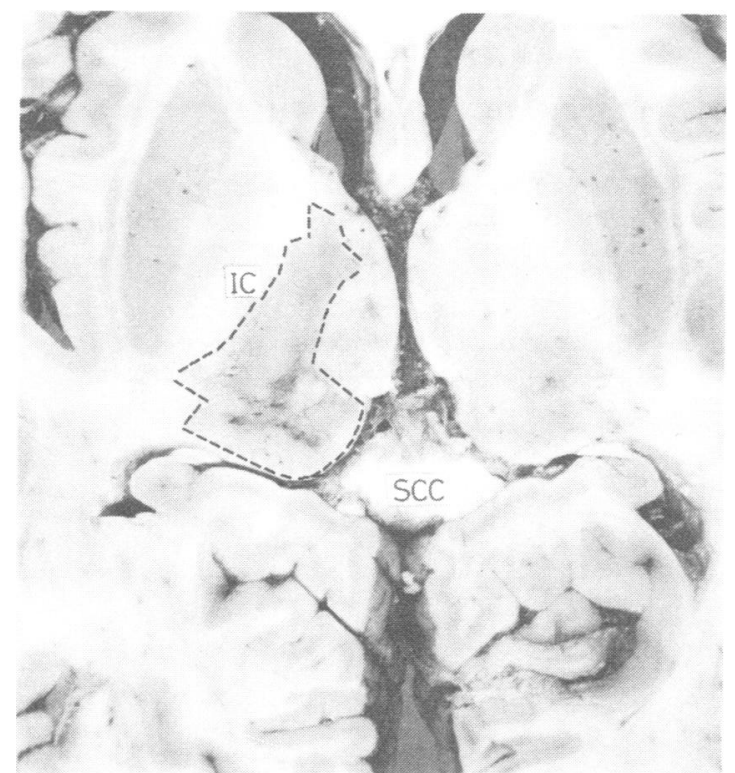

Fig 1 Horizontal section through mid-thalamus, demonstrating infarction (outlined) of left anterior, lateral, and posterior thalamus. IC: internal capsule, SCC: splenium of corpus collosum.

and was isolated to medial aspect of the genu and posterior limb. No pathology at more rostral levels or below the thalamus was present.

Electrophysiological recordings A 16 channel electroencephalogram was obtained in the waking state. Somatosensory evoked potentials were recorded in response to stimulation ( $0.2 \mathrm{~ms}$ pulses) of the left and right median nerve at the wrist. The stimulus level was just below the motor threshold for the thenar muscles. The stimulus rate was 3 per second. Short latency components were recorded from two locations, from scalp location $\mathrm{C}_{\mathrm{z}}$ and from over the seventh cervical vertebra. For both of these sites the reference electrode was placed on the dorsum of the unstimulated hand. A ground electrode was placed on the stimulated limb. The amplifier bandpass was $32-1600 \mathrm{hz}$ ( $3 \mathrm{~dB}$ down points). For the middle latency (15-60 ms) components the active electrodes were placed at P7 (P8) and F3 (F4) referred to the contralateral earlobe. Stimulus level was as before, the rate was 1 per second. Amplifier bandpass was $3 \cdot 2-800 \mathrm{hz}$. Evoked potentials were photographed from an oscilloscope.

Auditory evoked potentials were recorded in response to binaural stimulation (clicks:60 dBSL) presented over headphones. Electrodes were placed at scalp locations C3 and $\mathrm{C} 4$ and referred to the seventh cervical vertebra. Middle latency (15-60 ms) components were recorded to stimulus rates of 5 per second with amplifier bandpass of $32-320 \mathrm{hz}$; long latency potentials $(60-300 \mathrm{~ms})$ were recorded at stimulus rates of $\mathbf{0 . 2}$ per second, with amplifier bandpass of $0 \cdot 32-80 \mathrm{hz}$.

\section{Results}

The electroencephalogram showed distinct monomorphic delta waves localised in the left frontocentral region. A clear alpha rhythm which was present on the right, was absent on the left (fig 2).

Recordings from the vertex disclosed three positive far-field median nerve-evoked potential components with peak latencies of $9 \cdot 0,12 \cdot 5$, and $15 \cdot 0 \mathrm{~ms}$ (fig $3 \mathrm{~A}$ ). These components were recorded in response to both left and right median nerve stimulation. Left and right median nerve stimulation also evoked a negative component recorded over the neck peaking at about $14 \mathrm{~ms}$ (fig $3 \mathrm{~A}$, bottom traces). In response to left median nerve stimulation a clear N22-P28 potential was recorded over the right somatosensory cortex; a frontal N33 component was also recorded on the right (fig 3B). In contrast, stimulation of the right median nerve evoked no potentials over the left somatosensory or motor cortex, even if the stimulus raised to twice the motor threshold (fig 3B).

With auditory stimulation no hemispheric asymmetries were noted in the mid-latency (" $\mathrm{N}_{\mathrm{a}}-\mathrm{P}_{\mathrm{a}}$ ") or long latency (" $\mathrm{N}_{1}-\mathrm{P}_{2}$ ") components ${ }^{8}$ (fig $3 \mathrm{C}$ ).

\section{Discussion}

At the time of the recording sessions neuroradiological findings had suggested a discrete thalamic lesion and although a major object of this study was a delineation of the sources of the short and mid-latency somatosensory evoked potentials (SLSEPs and MLSEPs), some pathophysiological correlations could also be made for the EEG. The EEG of our patient was characterised by a prominent left fronto-central delta rhythm and absence of the alpha rhythm on the left. The pathophysiology of delta activity in the human EEG is poorly understood. ${ }^{-11}$ Gloor and colleagues have recently showed in cats, ${ }^{12}$ that discrete thalamic lesions caused unilateral focal delta waves overlying the lesion. The frontal delta rhythm in our patient, therefore, was likely to have been caused by destruction of anterior, lateral, and dorsomedial nuclei and interruption of their projection fibres. The unilateral suppression of the alpha rhythm is similar to recordings in patients with anterior and mesial thalamic tumours presented by Jasper and Van Buren, ${ }^{13}$ who felt that the most critical projections necessary for maintenance of the alpha rhythm arose from these areas.

Although evoked potentials are widely used to study and assess the human somatosensory pathway, the actual neural sources of many components are not firmly established. With regard to the early 


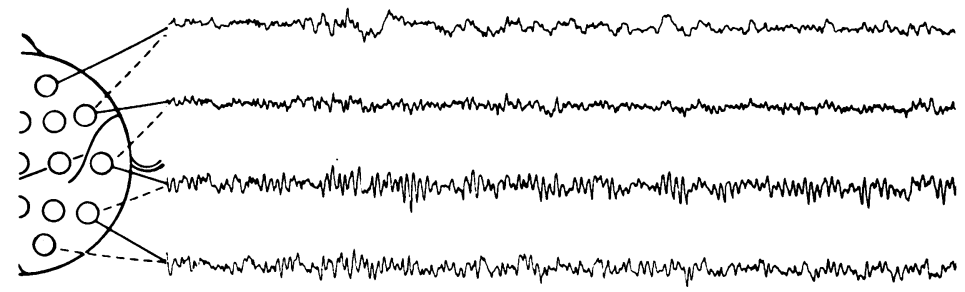

Fig 2 Electroencephalogram during waking state. Prominent

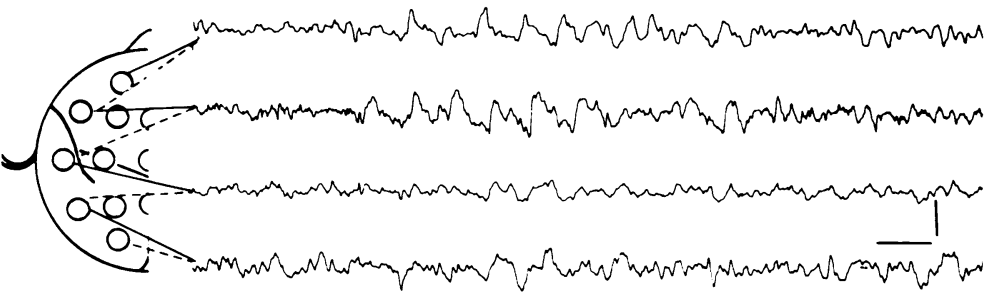
delta waves and suppression of the alpha rhythm on the left were recorded throughout the record. Calibrations: $1 \mathrm{~s}$ and $10 \mu \mathrm{V}$.

(a)

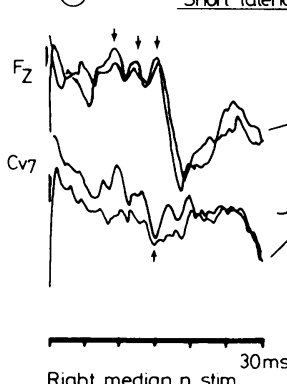

(b)
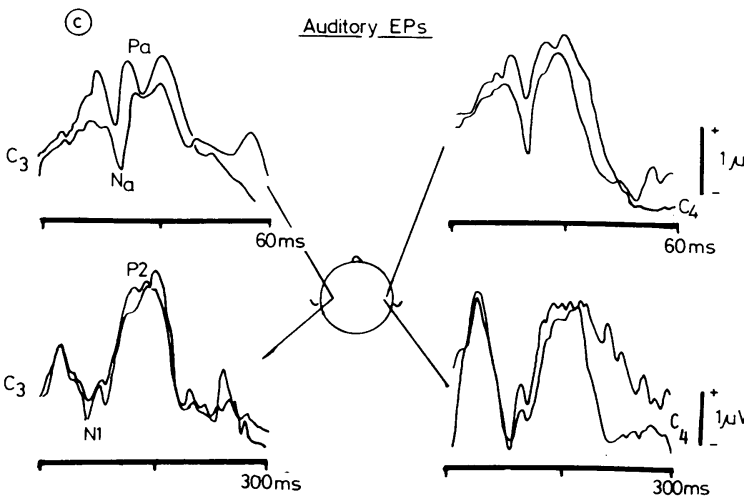

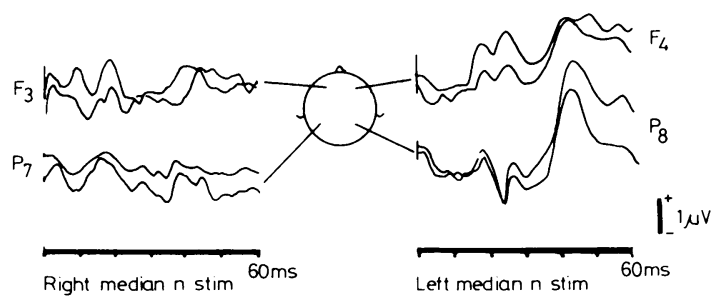

Fig 3 (A) Short latency somatosensory evoked potentials (median $n$ stimulation), recorded from scalp location $F_{z}$ and from over the seventh cervical vertebra; the reference ${ }_{v}$ electrode was on the unstimulated arm. Arrows indicate far field scalp positive waves and nuchal negativity. (B) Middle latency somatosensory evoked potentials (median $n$ stimulation) recorded from scalp locations $F_{3} P_{7} F_{4} P_{8}$ ( referred to $F_{z}$ ). Note absence of potentials over left hemisphere. (C) Middle latency and long latency auditory evoked potentials. (Binaural stimulation, $60 \mathrm{dBSL}$ ) recorded from $C_{3}$ and $C_{4}$ referred to seventh cervical vertebra. components, Mauguiere and Courjon have recently described ${ }^{7}$ two patients, one with a capsulo-thalamic haemorrhage and the other with a circatricial ischaemic lesion of the posterior thalamus (CT data); in both of these patients the SLSEPs could be recorded but the later MLSEPs were absent over the cortex ipsilateral to the lesion. Our patient who had an infarction involving the anterior and lateral thalamic nuclei also presented with intact SLSEPs but absent MLSEPs over the affected hemisphere indicating that the SLSEPs occurring within $15 \mathrm{~ms}$ post-stimulus are generated at a site caudal to the thalamus.

The exact neural source of the $\mathrm{N} 20$ of the MLSEPs is unknown; its generator is probably from within the primary somatosensory cortex or from 
the presynaptic thalamocortical fibres. Chiappa et $a l,{ }^{2}$ however, have presented CT data from a patient with a unilateral centrum semiovale leucoencephalopathy disconnecting the thalamus from the cortex, and have found that the N20 to be intact, suggesting that this potential is generated from within the thalamus. The lesion in our patient was restricted to the thalamus and all parietal and frontal MLSEPs were absent ipsilateral to the lesion, indicating that the thalamus is necessary for generation of N20 and succeeding components, but not required for components with a latency of less than $20 \mathrm{~ms}$. The intact middle and long latency auditory potentials in the patient reflect lack of damage to the medial geniculate and medial parts of the thalamus which are thought to be necessary for their generation.

The authors thank Shirley V Dunbar for assistance.

\section{References}

${ }^{1}$ Giblin DR. Somatosensory evoked potentials in healthy subjects and in patients with lesions of the nervous system. Ann NY Acad Sci 1964;112:93-142.

${ }^{2}$ Chiappa K, Choi SK, Young RR. Short latency somatosensory evoked potentials following median nerve stimulation in patients with neurological lesions. In: Desmedt JE, ed. Progress in Clinical Neurophysiology. Vol 7, Clinical Uses of Cerebral, Brainstem and Spinal Somatosensory Evoked Potentials. Basel: Karger, 1980:264-81.

${ }^{3}$ Desmedt JE, Cheron G. Somatosensory evoked potentials to finger stimulation in healthy octogenarians and young adults: Waveforms, scalp topography and transit times of parietal and frontal components. Electroencephalogr Clin Neurophysiol 1980;50:404-25.
4 Jones SJ. Short latency potentials recorded from the neck and scalp following median nerve stimulation in man. Electroencephalogr Clin Neurophysiol 1977; 43:853-63.

neck and scalp following median nerve stimulation in man. Electroencephalogr Clin Neurophysiol 1977;43:853-63.

${ }^{5}$ El-Negamy E, Sedgwick EM. Properties of a spinal somatosensory evoked potential recorded in man. $J$ Neurol Neurosurg Psychiatry 1978;41:762-8.

6 Desmedt JE, Cheron G. Central somatosensory conduction in man: Neural generators and interpeak latencies of the far field components recorded from neck and right and left scalp and earlobes. Electroencephalogr Clin Neurophysiol 1980;50:382-403.

${ }^{7}$ Mauguière F, Courjon J. The origins of short-latency somatosensory evoked potentials in humans. Ann Neurol 1981;9:607-11.

8 Picton TW, Hillyard SA, Krausz HI, Galambos R. Human auditory evoked potentials I. Evaluation of components. Electroencephalogr Clin Neurophysiol 1974;36:179-90.

${ }^{9}$ Cordeau JP. Monorhythmic frontal delta activity in the human electroencephalogram: A study of 100 cases. Electroencephalogr Clin Neurophysiol 1959;11:73346.

${ }^{10}$ Bagchi BK, Kooi KA, Selving BT. Subtentorial tumors and other lesions: An electroencephalographic study of 121 cases. Electroencephalogr Clin Neurophysiol 1961;13:180-92.

${ }^{11}$ Van der Drift JHA, Magnus O. Primary thalamic lesions. Electroencephalogr Clin Neurophysiol 1961;Suppl 19:125-37.

${ }^{12}$ Gloor P, Ball G, Schaul N. Brain lesions that produce delta waves in the EEG. Neurology (Minneap) 1977;27:326-33.

${ }^{13}$ Jasper H. Van Buren. Interrelationship between cortex and subcortical structures: Clinical electroencephalographic studies. Electroencephalogr Clin Neurophysiol 1953;Suppl 4:168-201. 